Research Article

\title{
State Identification of ECS Turbine Bearing Based on Fractal Dimension
}

\author{
Chenchen Li, ${ }^{1}$ Qingpeng Han ${ }^{D},{ }^{1}$ Rui Zhu, ${ }^{1}$ and Qingyu $\mathrm{Zhu}^{2}$ \\ ${ }^{1}$ College of Energy and Mechanical Engineering, Shanghai University of Electric Power, Shanghai 200090, China \\ ${ }^{2}$ School of Mechanical Engineering, Dalian University of Technology, Dalian 116024, China \\ Correspondence should be addressed to Qingpeng Han; han1011@163.com
}

Received 21 August 2018; Accepted 23 October 2018; Published 2 December 2018

Academic Editor: Konstantin Avramov

Copyright (c) 2018 Chenchen Li et al. This is an open access article distributed under the Creative Commons Attribution License, which permits unrestricted use, distribution, and reproduction in any medium, provided the original work is properly cited.

According to nonlinear characteristics of vibration signals measured on the turbine used in the aircraft environment control system (ECS), the ensemble empirical mode decomposition (EEMD) together with fractal dimension analysis is investigated in the paper to extract characteristic quantities for the goal of fault diagnosis of turbine bearings. Firstly, in order to filter noise signal vibration and advance signal-to-noise signals under different statements of bearings, including normal bearing, inner ring fault, outer ring fault, and cage fault, are decomposed by EEMD. Then correlation dimension of those signals phase is calculated, contrasted, and analyzed after space reconstruction. The experimental result shows that the correlation dimension, as nonlinear geometric invariants, can be used as the characteristic quantity of ECS turbine bearing on running state. Moreover, this method can accurately and effectively identify the running state of the bearing.

\section{Introduction}

The environmental control system (ECS) of the aircraft can ensure the comfort of the plane cabin and the safety of the whole flight process [1]. Bearing, as the key component of the ECS turbine, plays a vital role in the normal operation of the whole system. The vibration signals produced in the bearing operation contain a lot of information. The running state of the bearing can be learned through the extraction and analysis of the eigenvalues of the signals. It further predicts the trend of state change, discovers, and eliminates trouble in time.

Due to the large number of excitation sources in the working environment of bearings, the vibration signals are strongly nonlinear and nonstationary. The traditional classical signal processing method including time domain, frequency domain, and time-frequency domain analysis take time domain eigenvalue, such as root-mean-square, peak value, and so on and Fourier transform as the foundation mainly [2-5]. All of the methods assuming that the signal is stationary and linear are evidently not in conformity with the reality, which produce errors. To solve the problem, the theory of nonlinear dynamics like fractal theory has been developed rapidly. Fractal dimension, which is widely used in quantitative description of nonlinear system behavior, can quantitatively characterize chaotic attractors [6] because different running conditions can cause different geometric structures of vibration signals, which is in favour of distinguishing bearing states [7]. Some articles have demonstrated the validity of fractal theory in instrument state recognition [8-10].

Before analysis of the fractal dimension, it is necessary to preprocess the original signal to reduce the adverse effect of noise signal. In this research, a novel feature extraction method based on ensemble empirical model decomposition (EEMD) and correlation dimension is proposed and its application for rolling bearing fault diagnosis is described. Firstly, the original vibration signals decomposed into a series of intrinsic mode function (IMF) that contain the characteristics of the signal are selected to calculate its correlation dimension. Finally, the bearing state is judged according to the trend of dimension change. 


\section{Ensemble Empirical Mode Decomposition}

In 1998, Huang [11] et al. proposed a data-driven adaptive decomposition, empirical mode decomposition (EMD) method, which greatly improved the analysis effect of the nonlinear and nonstationary signal. However, in practical applications, there is a general problem of model mixing that is a single IMF containing the characteristic signal with maximum frequency difference or a signal with similar frequencies decomposed into different IMF in the method. The main reason for the model aliasing is that the abnormal events in the signal have an adverse effect on the selection of the extreme points which results in the uneven distribution of the extreme points to bring about the phenomenon of "overshoot" and "undershoot" in EMD process. To solve this problem, $\mathrm{Wu}$ [12] et al. put forward the method of EEMD. The method is to add the Gauss white noise of the finite amplitude to the decomposed signal and use the characteristic of Gauss white noise in the time frequency domain to smoothen the abnormal events, so as to reduce the adverse effects of abnormal events on the extreme point selection in the EMD process to achieve the purpose of the distribution of the extreme value point of the uniform signal. Finally, the white noise zero mean value is used to cancel the noise repeatedly.

The EEMD decomposition steps are summarized as follows:

(1) Gauss white noise $n_{i}(t)$ with a mean value of 0 and a constant amplitude standard deviation is added $\mathrm{N}$ times in the original signal $x(t)$, respectively:

$$
x_{i}(t)=x(t)+n_{i}(t)
$$

where $i=1 \sim N$.

(2) EMD decomposition of $x_{i}(t)$ is carried out, respectively. K IMF components and a remainder $r_{i}(t)$ are obtained at each decomposition:

$$
x_{i}(t)=\sum_{j=1}^{K} c_{i j}(t)+r_{i}(t) \text {. }
$$

Among which, $c_{i j}(t)$ is the $j$-th IMF obtained through EMD decomposition after the $i$-th adding Gauss white noise, $j=1 \sim N$.

(3) According to the principle that the mean value of the uncorrelated random sequence is 0 , the total average operation of the IMF corresponding to the above steps is used to eliminate the effect of repeated Gauss white noise on real IMF. Then, the IMF and the remainder $r(t)$ after the EEMD decomposition are obtained:

$$
\begin{gathered}
c_{j}(t)=\frac{1}{N} \sum_{i=1}^{N} c_{i j}(t), \\
r(t)=\frac{1}{N} \sum_{i=1}^{N} r_{i}(t),
\end{gathered}
$$

where $c_{j}(t)$ is the $j$-th IMF obtained through EEMD decomposition of original signal.

Finally, $K$ IMF components and a remainder $r(t)$ are gained:

$$
x(t)=\sum_{j=1}^{K} c_{j}(t)+r(t) .
$$

In order to verify the effectiveness of EEMD for overcoming model aliasing, a high frequency pulse signal was added to a low frequency sine signal to synthesize a simulation signal as shown in Figure 1.

Then, the simulation signal was decomposed by EMD and EEMD, respectively, to get a series of IMF components as shown in Figures 2 and 3. It can be clearly seen from Figure 2 that model mixing was caused by EMD decomposition because not only $\mathrm{c} 1$ but also $\mathrm{c} 2$ contained both the pulse signal and the sinusoidal signal. At the same time, the sinusoidal signal was decomposed into different IMF, which made the decomposition result lose the physical meaning seriously and not reflecting the essence of the signal. In contrast, the average number of times in the EEMD decomposition process was 50 times, and the added Gauss white noise amplitude was 0.02 times the standard deviation of the original signal. It can be clearly seen from Figure 3 that the decomposition result avoided the model mixing effectively in which the pulse signal decomposed into the $\mathrm{c} 1$, and the sinusoidal signal decomposed into the $\mathrm{c} 3$ and the $\mathrm{c} 2$ caused by the signal modulation.

\section{Correlation Dimension}

Dimension refers to the number of independent coordinates used to describe the location of a point in space. In classical geometry, the dimensions are all integers, while the fractal theory extends the dimension to the fractions. These fractions are called fractal dimensions. As a fractal dimension, correlation dimension is very sensitive to the change of the attractor's mechanism and can effectively reflect the nonlinear behavior of the system.

The G-P algorithm is an effective method for calculating the correlation dimension of the system according to time series based on the embedding theorem and the phase space reconstruction theory, which mainly consists of two steps: phase space reconstruction and correlation dimension calculation.

3.1. Phase Space Reconstruction. Phase space reconstruction, proposed by $\mathrm{R}$ Mane and $\mathrm{F}$ Takens, is the delay embedding principle which constructs the one-dimensional time $\left\{x_{i}\right\}=$ $\left\{x_{1}, x_{2}, \cdots, x_{N}\right\}$ into multidimensional phase space to show the parting characteristics of the internal structure of the data. The reconstructed phase space can be shown as follows:

$$
X_{n \times m}=\left[\begin{array}{cccc}
x_{1} & x_{1+\tau} & \cdots & x_{1+(m-1) \tau} \\
x_{2} & x_{2+\tau} & \cdots & x_{2+(m-1) \tau} \\
\vdots & \vdots & \ddots & \vdots \\
x_{n} & x_{n+\tau} & \cdots & x_{n+(m-1) \tau}
\end{array}\right]=\left[\begin{array}{c}
X_{1} \\
X_{2} \\
\vdots \\
X_{n}
\end{array}\right],
$$

where $N$ is the number of time series, $n$ is the number of vectors of reconstructed phase space and $n=N-(m-1) \tau$, $m$ is embedding dimension, and $\tau$ is embedded time delay parameter, integer times of sampling time interval $\Delta t$. 

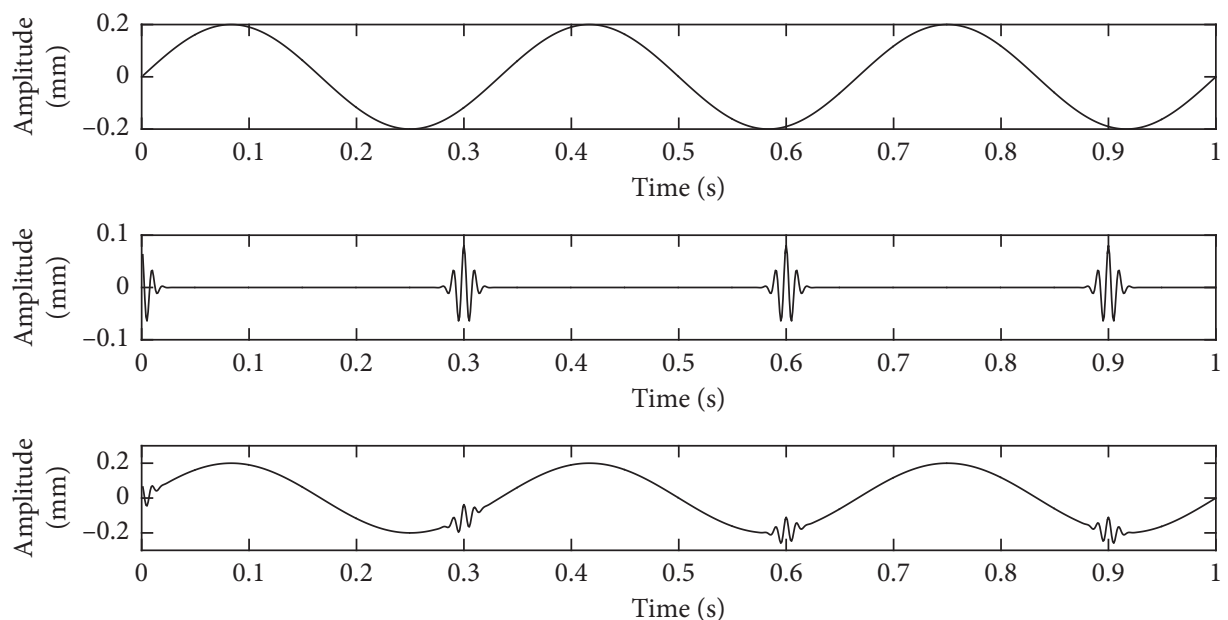

Figure 1: Simulation signal.
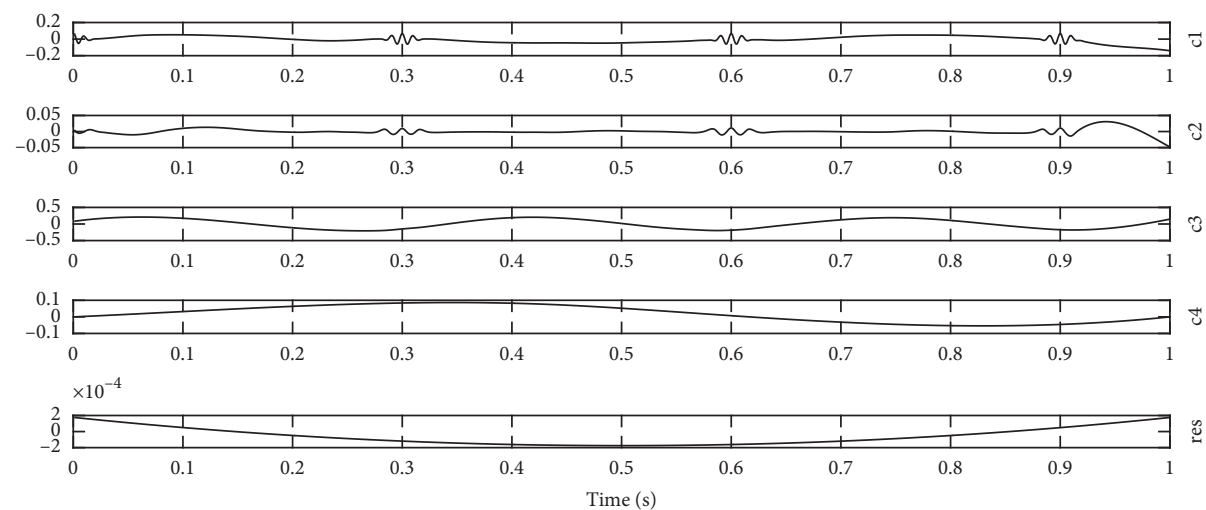

Figure 2: EMD decomposition results.
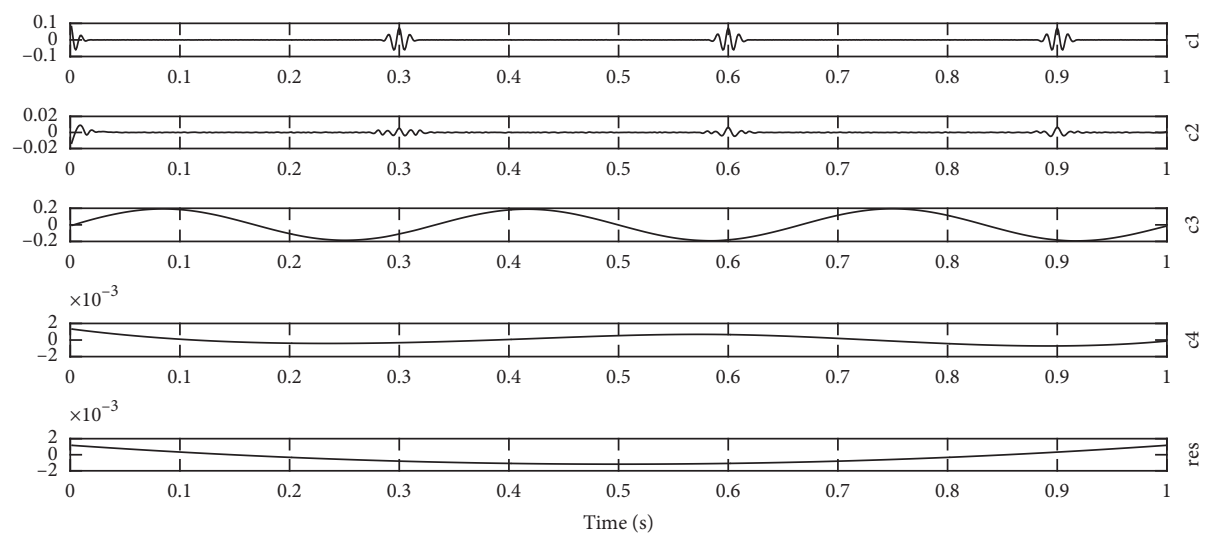

Figure 3: EEMD decomposition results.

3.2. Correlation Dimension Calculation. In the reconstructed phase space $X_{n \times m}$, the space distance between any two points is calculated by using vectors $X_{i}$ as points:

$$
S_{i j}=\left|X_{i}-X_{j}\right|=\sqrt{\sum_{k=0}^{m-1}\left(x_{i+k \tau}-x_{j+k \tau}\right)^{2}},
$$

where $i, j=1,2, \cdots, n$. The distance between any two points in the phase space is reconstructed to form the following matrix:

$$
S_{n \times n}=\left[\begin{array}{cccc}
s_{11} & s_{12} & \cdots & s_{1 n} \\
s_{21} & s_{22} & \cdots & s_{2 n} \\
\vdots & \vdots & \ddots & \vdots \\
s_{n 1} & s_{n 2} & \cdots & s_{n n}
\end{array}\right] .
$$




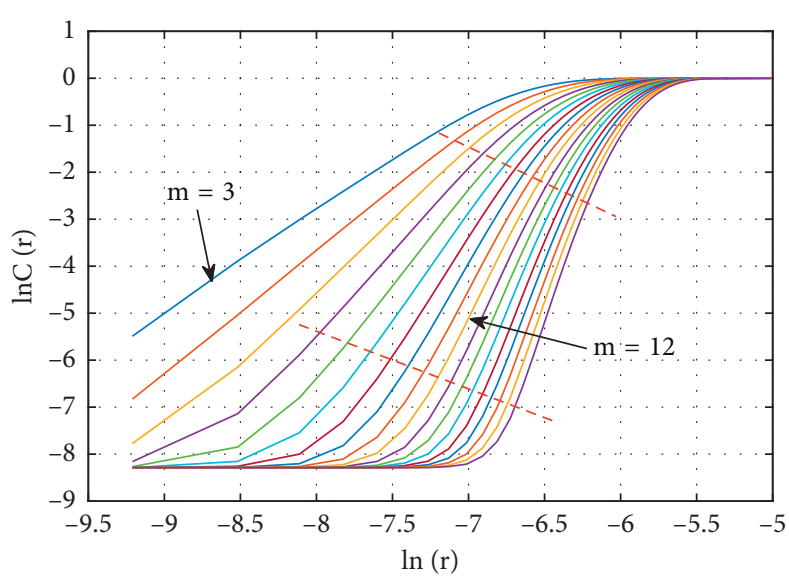

(a)

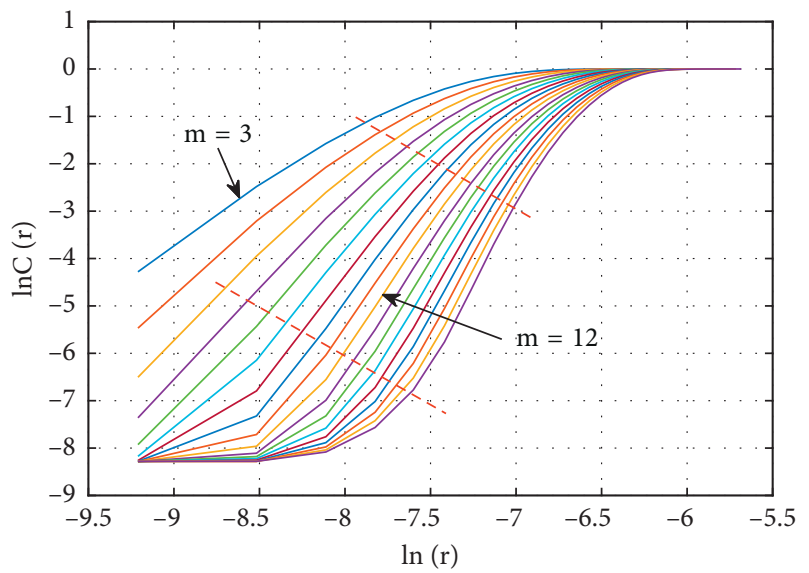

(c)

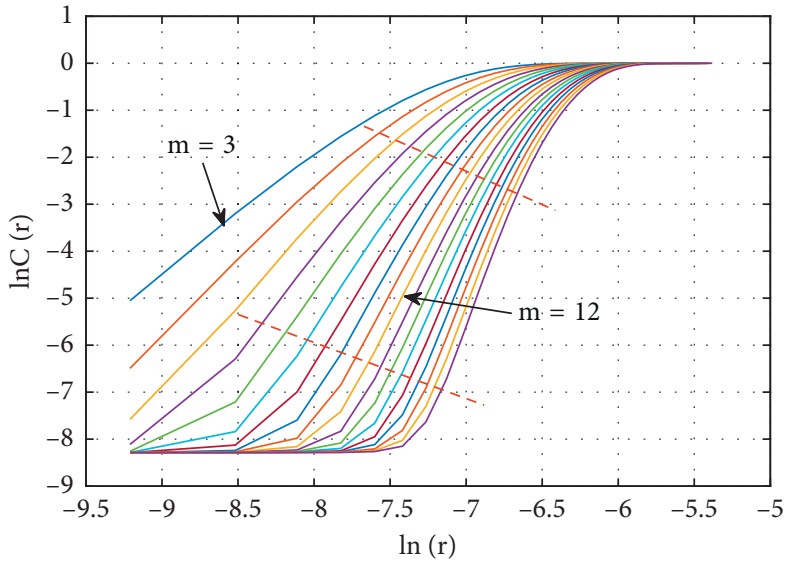

(b)

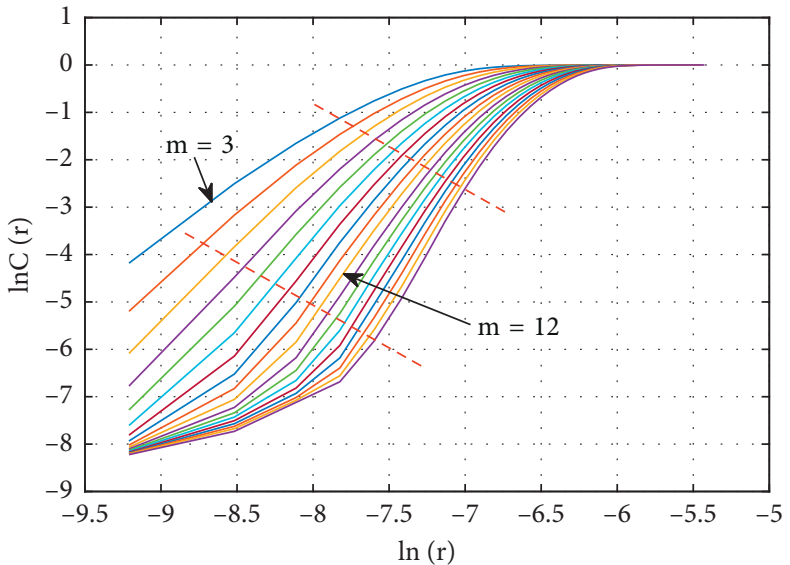

(d)

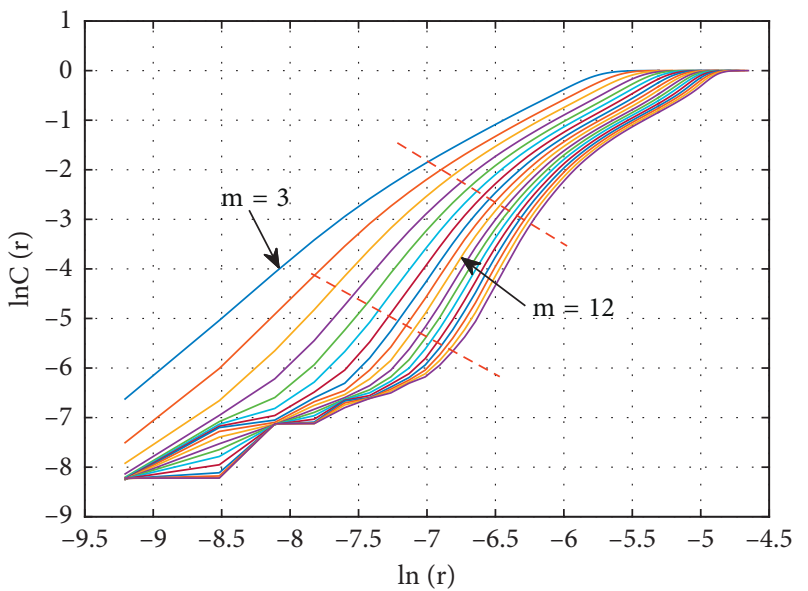

(e)

FIgURE 4: The correlation integral map of 5 IMFs under normal condition. (a) IMF1. (b) IMF2. (c) IMF3. (d) IMF4. (e) IMF5.

The matrix is a symmetric matrix of 0 diagonal lines. The real number $r$ is in the interval $\left[s_{\min }, s_{\max }\right]$ in which $s_{\max }$ is the maximum of the matrix and $s_{\min }$ is minimum. The ratio of the number of elements in the matrix smaller than $r$ to the total number of elements is recorded as follows:

$$
C(r)=\frac{1}{n^{2}} \sum_{i, j=1}^{n} H\left(r-s_{i j}\right)
$$

where $H\left(r-s_{i j}\right)$ is unit step function. 


$$
H\left(r-s_{i j}\right)= \begin{cases}1, & r \geq s_{i j} \\ 0, & r<s_{i j}\end{cases}
$$

The value of $r$ is changed arbitrarily in the interval $\left[s_{\min }, s_{\max }\right]$ to get the corresponding value $C(r)$ and make a double logarithmic coordinate curve $\ln C(r)-\ln (r)$. The interval of a better linear relationship in the curve is called scale-free region, whose slope is the correlation dimension $D$ of one-dimension time series:

$$
D=\lim _{r \longrightarrow 0} \frac{\ln C(r)}{\ln (r)} .
$$

3.3. Selection of Key Parameters. In the whole calculation process, there are three main parameters: time delay $\tau$, embedding dimension $m$, and distance reference value $r$ to consider.

Time delay can separate signal data to expose its internal fractal characteristics. If it is too big, the correlation of data will become weaker, which causes the reconstructed time series not reflecting the overall information of the system. If it is too small, the correlation of data will become redundant, which causes the information of the system not expose easily. This paper used autocorrelation function to determine the value of time delay $\tau$.

The autocorrelation function of vibration signal time series $\left\{x_{i}\right\}$ is defined as follows:

$$
R(\tau)=\frac{1}{N-\tau} \sum_{i=1}^{N-\tau} x(i) x(i+\tau),
$$

where $R(0)$ is the largest. When $R(\tau)$ is reduced to $1-1 / e$ times of $R(0)$ for the first time, $\tau$ is the best delay for phase space reconstruction.

Embedding dimension $m$ refers to the minimum phase space series that can completely contain attractors of state transition. In 1980, Takens proved that it should be greater than $2 d+1$, where $d$ is the dimension of the space in which the attractor of the original state space. Meanwhile, when $r$ tends to 0 , the selection of $m$ should make the correlation dimension tending to be stable. If the embedding dimension is too small, the reconstructed phase space can not reflect the dynamic characteristics of the original system. If the embedding dimension is too large, the amount of computation will increase, while the excess phase space dimension will enlarge the noise effect of the original time series.

In this paper, the embedding dimension $m$ is 3 and gradually increased to 20 . The phase space of the bearing vibration signal is reconstructed, respectively, and the correlation integral map of 5 IMFs under normal condition, shown in Figure 4, with different embedding dimensions is made.

As we can see from the graph, there is a strongly linear scale-free region on the curve between the two red dotted lines. When the $m$ value is small, the slope of the scale-free zone is small and the interval is large. With the increase of $m$, the slope is also increasing, the interval decreases, and finally reaches saturation state. At the time $m>12$, the straight line of scale-free zone tended to be parallel, that is,

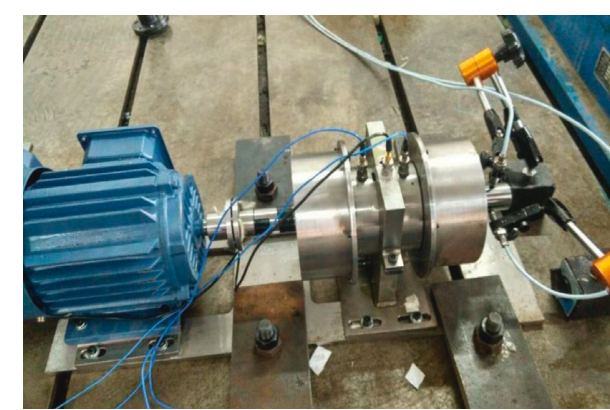

FIgURE 5: Experimental platform.

the correlation dimension tended to be stable. Overall consideration, the $m$ of this experimental data is 12 which is more reasonable. Other working conditions are similar.

It can be obtained by Formula (10) that the distance reference value $r$ directly affects the correlation dimension. If $r$ is too small, the number of the distance of two points in the reconstructed phase space is too less. $C(r)$ is close to 0 , which can not reflect the internal relationship of signal data. On the contrary, if $r$ is very large, $C(r)$ is close to 1 , which is also of no practical significance.

\section{Experimental Analysis}

The experimental platform consisted of rotor system and data acquisition system as shown in Figure 5. The rotor system was composed of the driving system module, the spindle system module, the tooling module, and the lubricating system module, which can simulate the characteristics of the actual ring control system. The driving system module is composed of an asynchronous motor with rated speed of $2800 \mathrm{r} / \mathrm{min}$ and rated power of $750 \mathrm{~W}$. The rolling bearings of four different working conditions (normal condition, inner ring fault, outer ring fault, and cage split) were placed in the specimen module, and the vibration signals were measured by eddy current sensor with a sensitivity of $1.3 \mathrm{~V} / \mathrm{mm}$.

The inner ring fault and outer ring fault were all $2 \mathrm{~mm}$ width scratches by spark cutting. The speed of the motor is $2400 \mathrm{r} / \mathrm{min}$. The sampling frequency of the data acquisition system is $4000 \mathrm{~Hz}$. One set of data were collected at each condition. The sampling time last for one second, which means there are 4000 values in each group. The time-domain of vibration signals under 4 working conditions are shown in Figure 6.

From Figure 6, we can see that a large number of vibration signals were generated when the bearings were running and eddy current sensors can accurately collect these signals. It is difficult for us to distinguish the working state of the bearing from the time domain diagram. Each group of vibration signal was decomposed by EEMD in order to calculate correlation dimension, and the results are shown in Figure 7.

Since EEMD decomposition is a principle component separation method, that is, the most important information contained in the original signal, it is extracted firstly. As can be seen from Figure 7, the main components of the vibration signal of the bearing are concentrated in the first 5 IMFs, and 


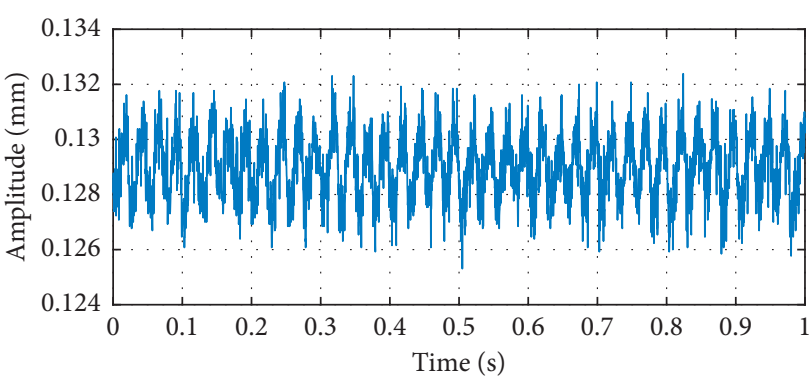

(a)

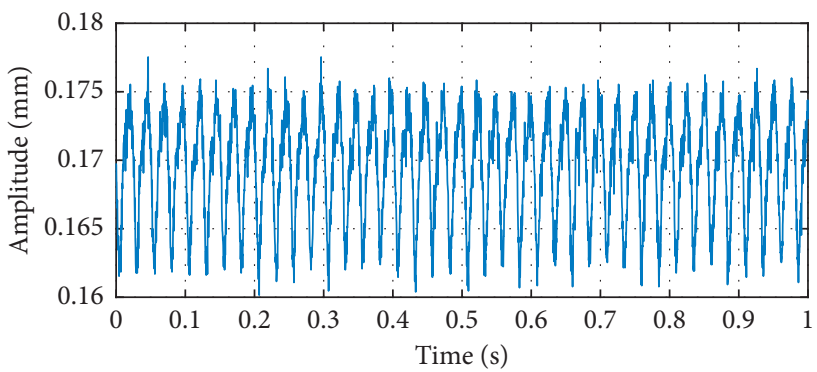

(c)

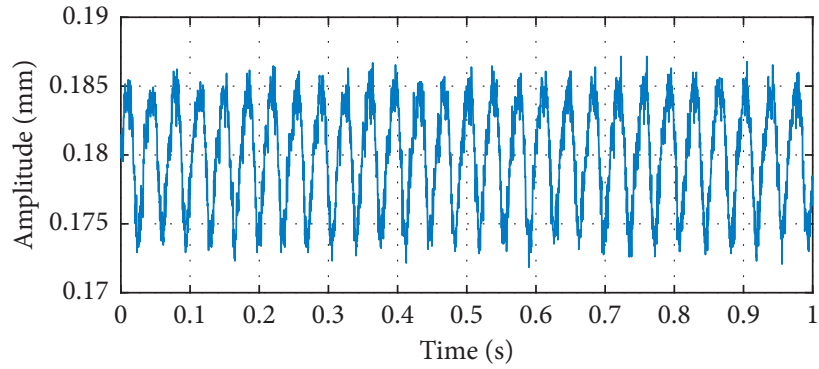

(b)

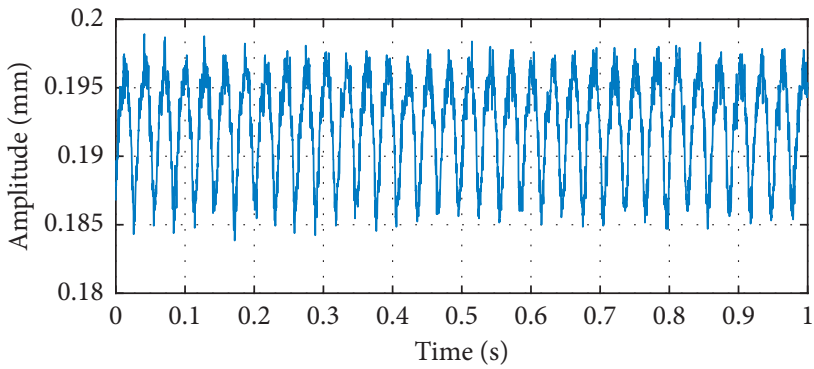

(d)

FIgURE 6: The time-domain of original vibration signals in 4 working states.

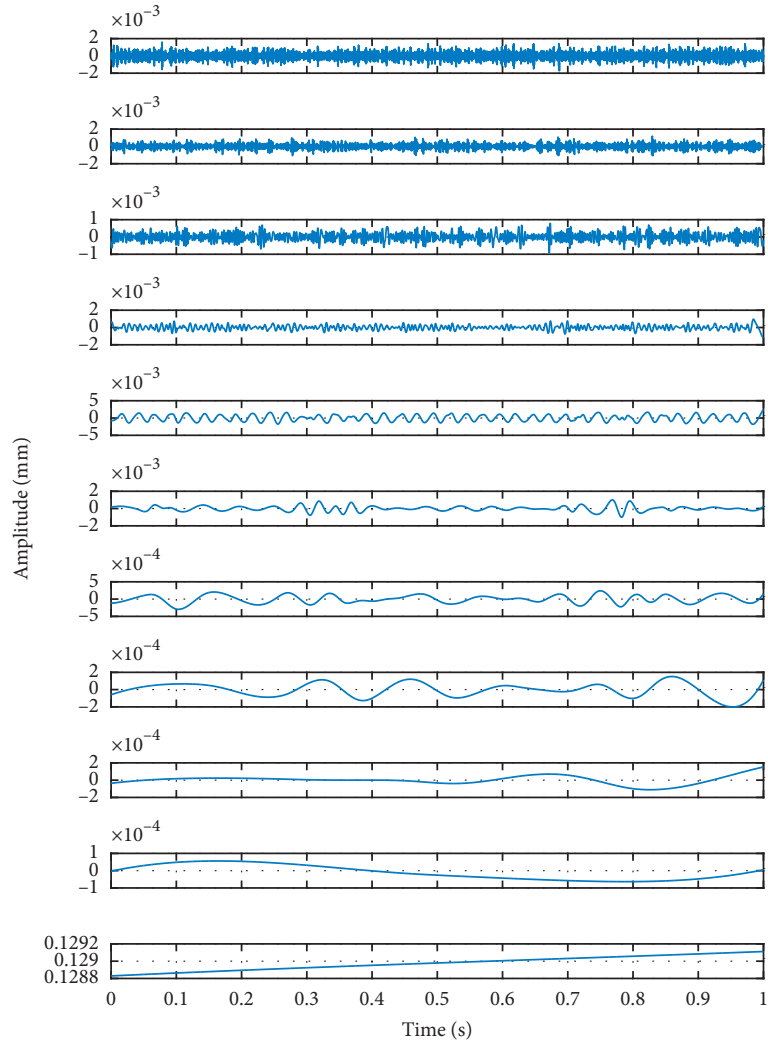

(a)

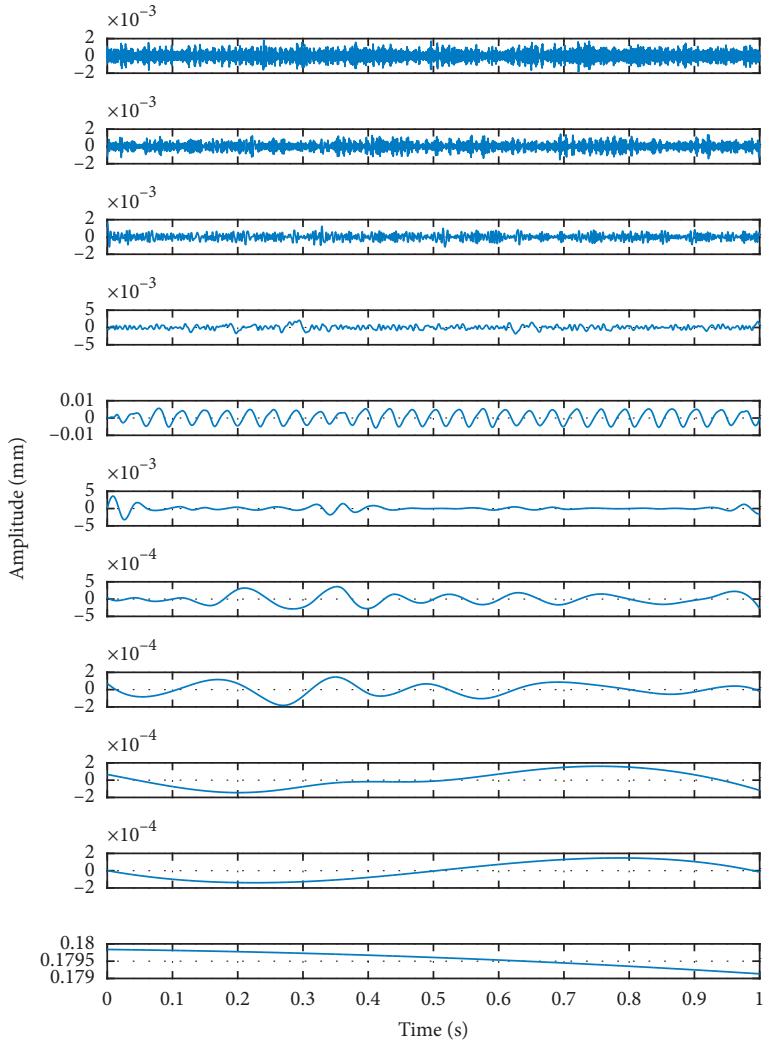

(b)

Figure 7: Continued. 


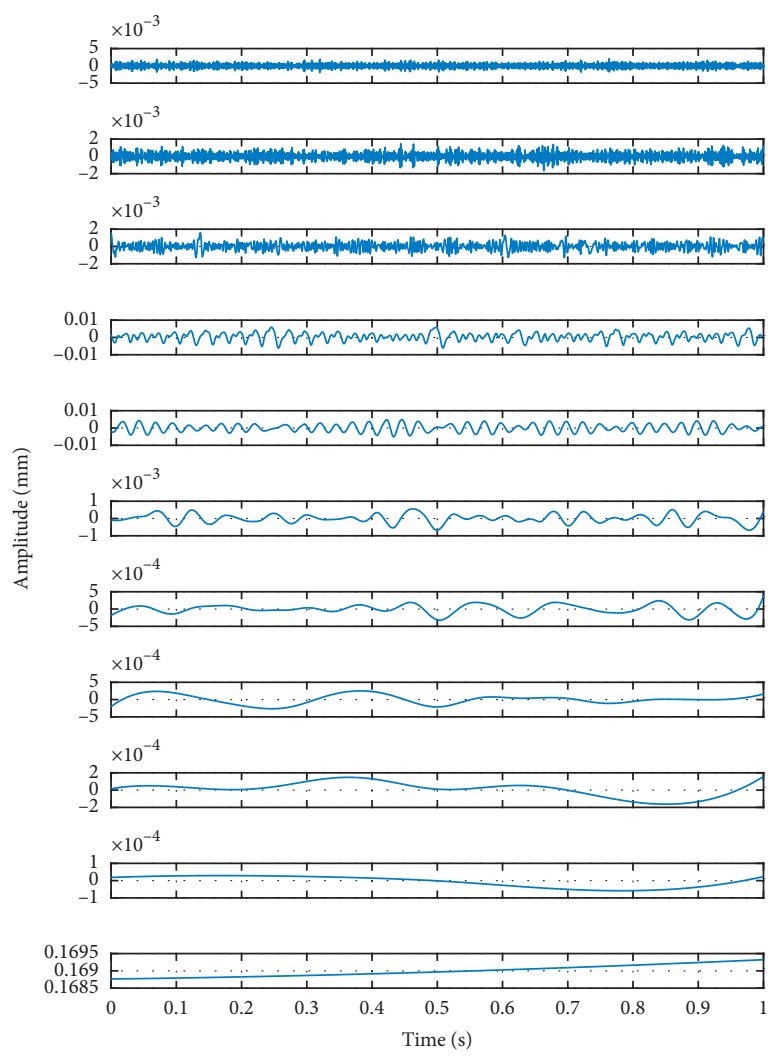

(c)

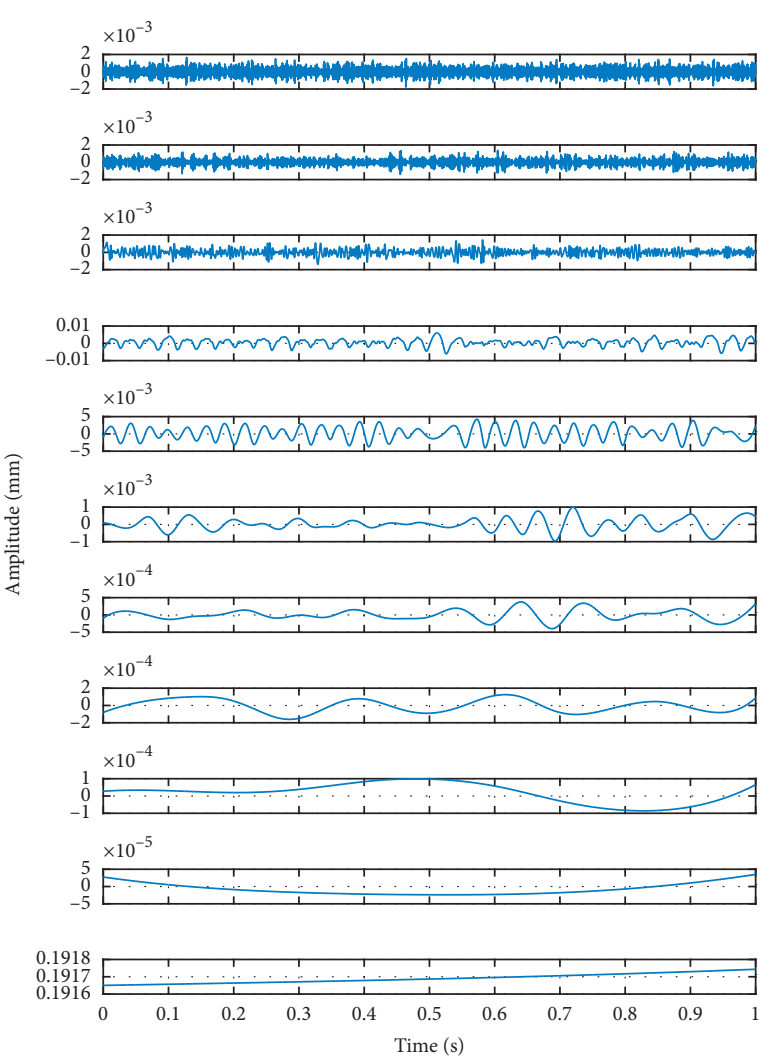

(d)

FIgURE 7: EEMD decomposition results of vibration signals in 4 working states. (a) Normal condition. (b) Outer ring fault. (c) Inner ring fault. (d) Cage split.

the environmental noise is separated to the low frequency part. The above correlation dimension algorithm was used to calculate these IMFs to distinguish different working states. The result is shown in Figure 8. It can be seen that the correlation dimension of IMF in four working states is different. Furthermore, the number decreases in turn with the modal decomposition of all signals except in case of outer ring fault, in which the dimension of fourth layers of IMF increases suddenly. This situation can be also seen from Figure 9, the correlation integral diagram of the fourth layer IMF.

In Figure 9, the dashed line 1 is approximate to the scalefree area under normal working sate, line 2 to the outer ring fault, and line 3 to the inner ring fault and the cage split. It is obvious that the slope of line 2 larger than that of the other two.

In Figure 8, the coincidence degree and trend of the signal under unknown condition and the signal in outer ring fault is higher than the other two states. Therefore, it is possible to determine the unknown condition as the outer ring fault, which also proves the feasibility of bearing fault diagnosis combined with EEMD and correlation dimension. The method is reasonable. Meanwhile, the slope of No. 2 dotted line is obviously greater than that of No. 1 and No. 3 . This phenomenon confirms the sudden increase of the correlation dimension of IMF4 in the outer ring fault signal in Figure 8.

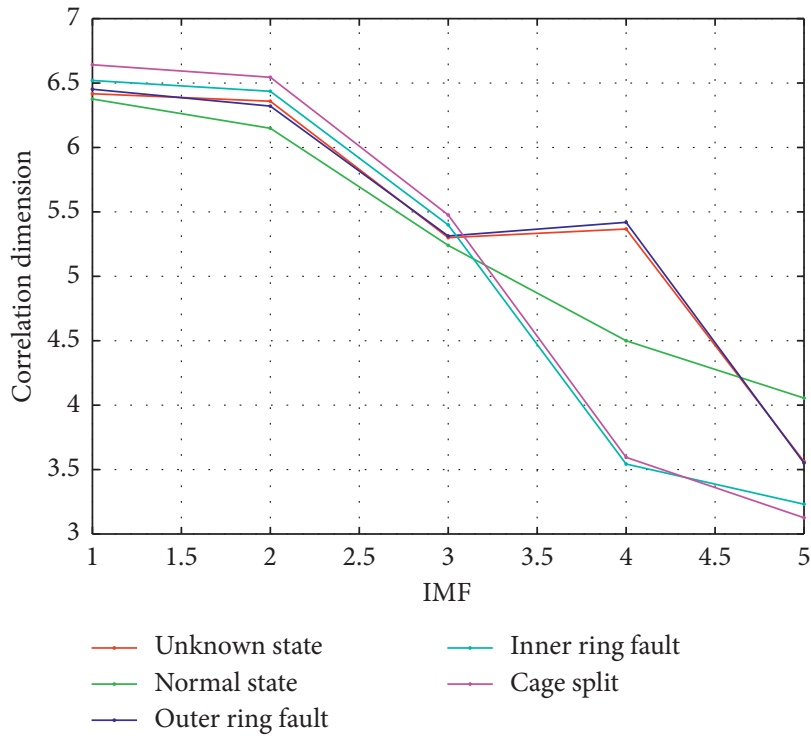

FIGURE 8: Correlation dimension curve.

\section{Conclusion}

The complexity of the vibration signal of the ECS turbine bearing under different working conditions is different. As a nonlinear analysis method, fractal theory is of general 


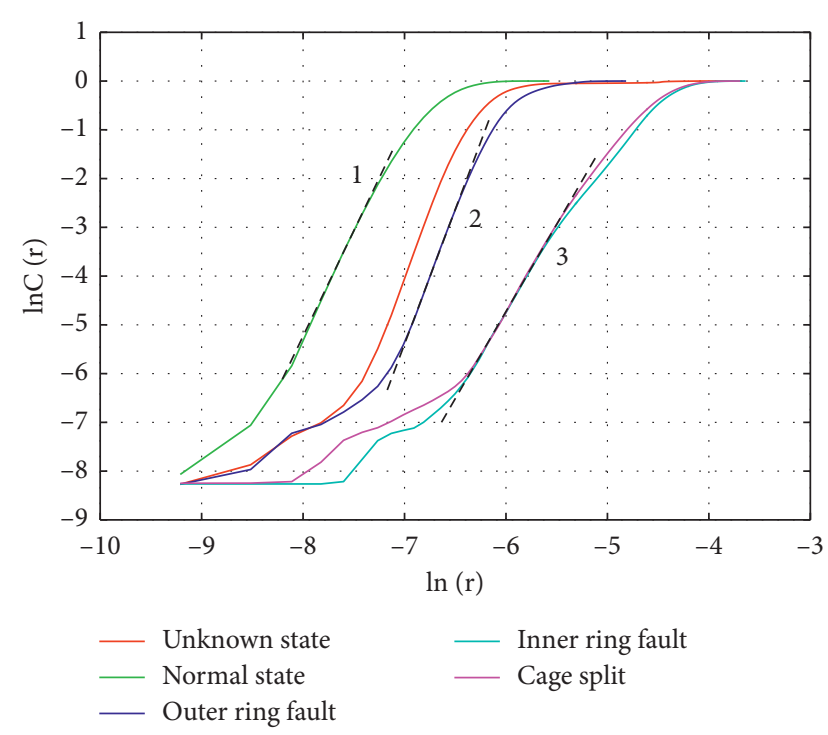

FIGURE 9: Fractal integral curve of IMF4.

significance in measuring the complexity of time series. Therefore, the fractal dimensions are very suitable for the analysis of various states of bearing vibration. Correlation dimension as a fractal dimension can be used to characterize the vibration signal. This paper uses EEMD instead of EMD to decompose the signal to avoid the appearance of modal aliasing. Then, the first 5 IMFs including the main components of the vibration signal are selected to draw the correlation dimension curve. The fault type of unknown signal can be accurately identified by comparing the coincidence degree and changing trend of the curve in the experiment. So this is a valuable method.

\section{Data Availability}

The data used to support the findings of this study are available from the corresponding author upon request.

\section{Conflicts of Interest}

The authors declare that they have no conflicts of interest.

\section{Acknowledgments}

This work is supported by the National Natural Science Foundation of China (No.11472068).

\section{References}

[1] M. Dechow and C. A. H. Nurcombe, Air Quality in Airplane Cabins and Similar Enclosed Spaces, Springer Berlin Heidelberg, Berlin, Heidelberg, 2005.

[2] H. Ocak, Fault Detection, Diagnosis and Prognosis of Rolling Element Bearings Frequency Domain Methods and Hidden Markov Modeling, Case Western Reserve University, Cleveland, OH, USA, 2004.

[3] H. Qiu, J. Lee, J. Lin et al., "Wavelet filter-based weak signature detection method and its application on rolling element bearing prognostics," Journal of Sound and Vibration, vol. 289, no. 4-5, pp. 1066-1090, 2006.
[4] A. K. S. Jardine, D. Lin, and D. Banjevic, "A review on machinery diagnostics and prognostics implementing conditionbased maintenance," Mechanical Systems and Signal Processing, vol. 20, no. 7, pp. 1483-1510, 2006.

[5] N. Tandon and A. Choudhury, "A review of vibration and acoustic measurement methods for the detection of defects in rolling element bearings," Tribology International, vol. 32, no. 8, pp. 469-480, 1999.

[6] M. Ahmadlou, H. Adeli, and A. Adeli, "Fractality and a wavelet-chaos-methodology for EEG-based diagnosis of Alzheimer disease," Alzheimer Disease and Associated Disorders, vol. 25, no. 1, p. 85, 2011.

[7] B. Li, P. L. Zhang, G. Q. Ren et al., "Mathematic morphologybased fractal dimension calculation and its application in fault diagnosis of roller bearings," Journal of Vibration and Shock, vol. 29, no. 5, pp. 191-194, 2010.

[8] J. P. Amezquitasanchez, M. Valtierrarodriguez, C. A. Perezramirez et al., "Fractal dimension and fuzzy logic systems for broken rotor bar detection in induction motors at start-up and steadystate regimes," Measurement Science and Technology, vol. 28, no. 7, article 075001, 2017.

[9] J. Li, H. Wei, L. Fan et al., "Multifractal detrended fluctuation analysis of frictional vibration signals in the running-in wear process," Tribology Letters, vol. 65, no. 2, p. 50, 2017.

[10] B. Wang, X. Hu, and H. Li, "Rolling bearing performance degradation condition recognition based on mathematical morphological fractal dimension and fuzzy C-means," Measurement, vol. 109, 2017.

[11] N. E. huang, Z. shen, and S. R. long, "The empirical mode decomposition and the Hilbert spectrum for nonlinear and non-stationary time series analysis," Proceedings Mathematical Physical and Engineering Sciences, vol. 454, no. 1971, pp. 903-995, 1998.

[12] Z. Wu and N. E. Huang, "Ensemble empirical mode decomposition: a noise-assisted data analysis method," Advances in Adaptive Data Analysis, vol. 1, no. 1, pp. 1-41, 2009. 


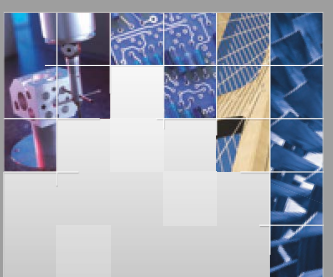

\section{Enfincering}
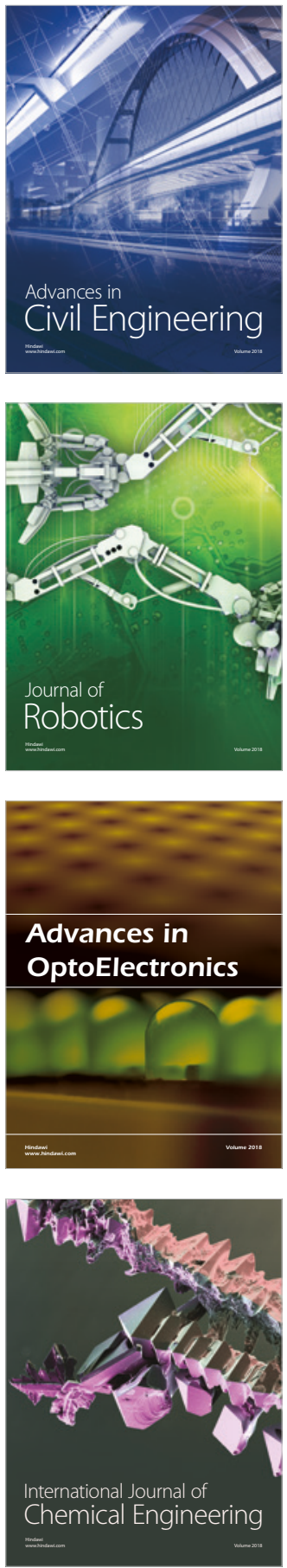

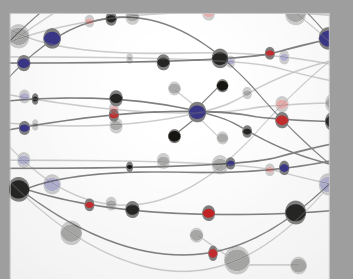

\section{Rotating \\ Machinery}

The Scientific World Journal

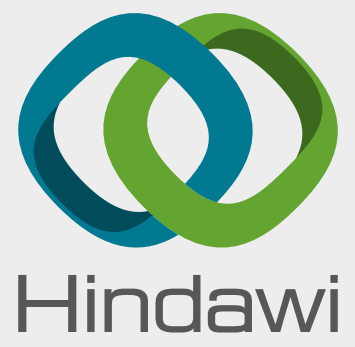

Submit your manuscripts at

www.hindawi.com
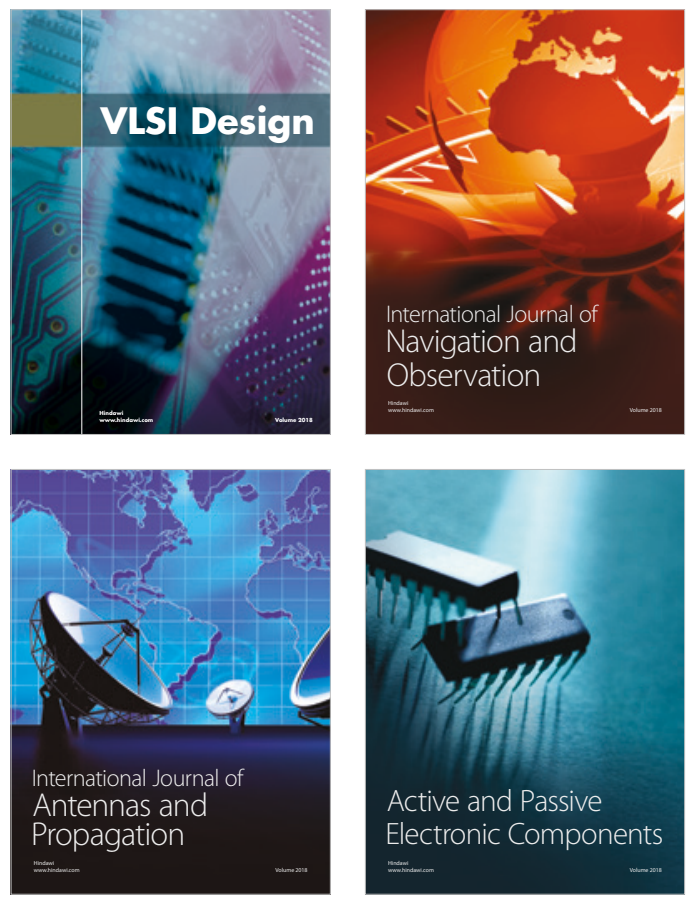
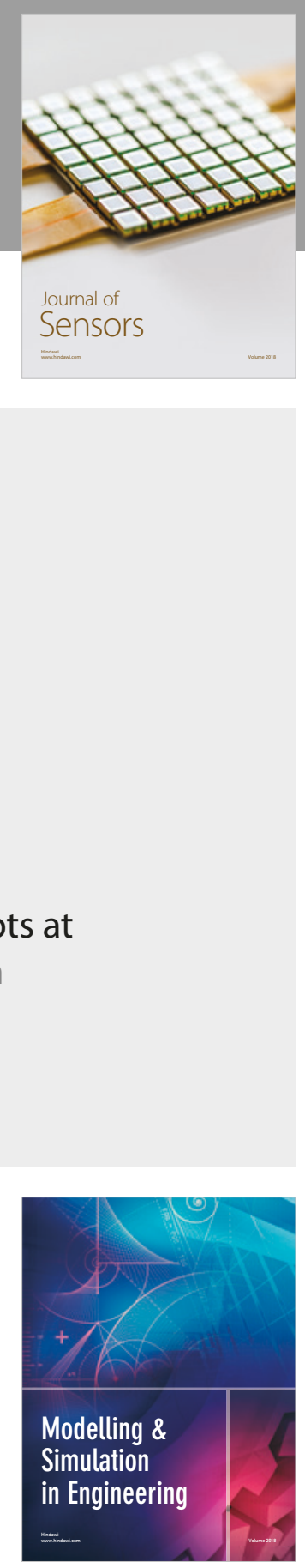

\section{Advances \\ Multimedia}
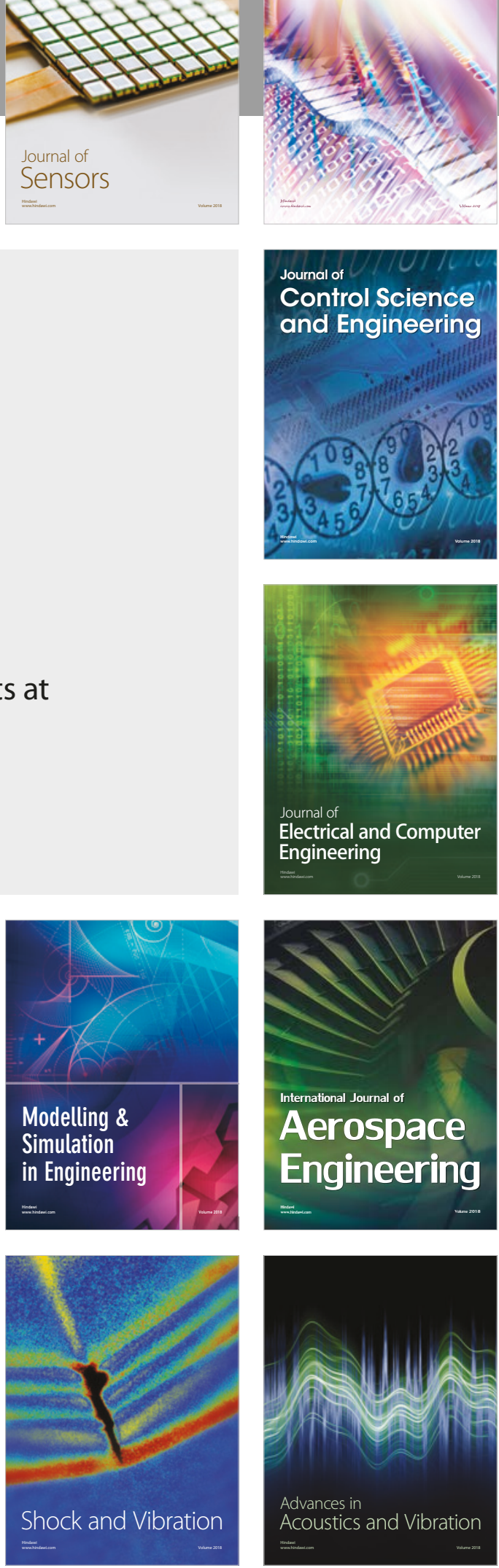